\title{
US adolescents' friendship networks and health risk behaviors: A systematic review of studies using social network analysis and Add Health data
}

Kwon Chan Jeon, Patricia Goodson

Background: Documented trends in health-related risk behaviors among US adolescents have remained high over time. Studies indicate relationships among mutual friends are a major influence on adolescents' risky behaviors. Social Network Analysis (SNA) can help understand friendship ties affecting individual adolescents' engagement in these behaviors. Moreover, a systematic literature review can synthesize findings from a range of studies using SNA, as well as assess these studies' methodological quality. Review findings also can help health educators and promoters develop more effective programs. Objective: This review systematically examined studies of the influence of friendship networks on adolescents' risk behaviors, which utilized SNA and the Add Health data (a nationally representative sample).

Methods: We employed the Matrix Method to synthesize and evaluate 15 published studies that met our inclusion and exclusion criteria, retrieved from the Add Health website and 3 major databases (Medline, Eric, and PsycINFO). Moreover, we assigned each study a methodological quality score (MQS).

Results: In all studies, friendship networks among adolescents promoted their risky behaviors, including drinking alcohol, smoking, sexual intercourse, and marijuana use. The average MQS was 4.6, an indicator of methodological rigor (scale: 1 - 9).

Conclusion: Better understanding of risky behaviors influenced by friends can be useful for health educators and promoters, as programs targeting friendships might be more effective. Additionally, the overall MQ of these reviewed studies was good, as average scores fell above the scale's mid-point. 
2

3

4

5

6

7
Department of Health and Kinesiology

Texas A\&M University

College Station, TX USA

Patricia Goodson, Ph.D.

Department of Health and Kinesiology

Texas A\&M University

College Station, TX USA

Corresponding Author

Kwon Chan Jeon, Ph.D.

4243 TAMU Blocker 316

College Station, TX 77843 USA

Phone: (979) 458-2217

Email: kcjeon@email.tamu.edu 
Introduction

The U.S. Youth Risk Behavior Survey (YRBS) has captured trends in health-related risk behaviors among adolescents in grades 9 to 12 between 1991-2011 (Centers for Disease Control and Prevention, 2012c). In the report, the number of adolescents who "had sexual intercourse with four or more persons (15.3\%)" (Centers for Disease Control and Prevention, 2012d), and "used chewing tobacco, snuff, or dip on school property on at least 1 day (7.7\%)" demonstrated a general increase (Centers for Disease Control and Prevention, 2012e). On the other hand, the number of those who "ever had at least one drink of alcohol on at least 1 day" showed a slight decrease from $72.5 \%$ to 70.8\% during that time (Centers for Disease Control and Prevention, 2012a).

Based on these data, we ask: "What may have shaped these trends over time?" In attempts to answer this question, researchers have indicated that risky health behaviors among adolescents are strongly influenced by their peers or friendship relationships (Hall \& Valente, 2007; Prinstein, Brechwald, \& Cohen, 2011; Rew \& Horner, 2003). Despite this knowledge, the literature on health risk behavior during adolescence has focused, traditionally, on individual adolescent risk taking behaviors (e.g., whether adolescents engaged in smoking, drinking or sexual activity, as well as frequency or intensity of engagement) as the unit of analysis. More recently, however, advanced analytical methodologies — including Social Network Analysis (SNA) - have led to the study of patterns in health risk behaviors influenced by peer or social contexts (e.g., friendship networks and affiliations).

Assessing patterns has highlighted the utility of SNA for in-depth understanding of the risky health behaviors among adolescents based on their relationships or interactions with other peers (Ennett et al., 2006; Haas, Schaefer, \& Kornienko, 2010). SNA is an optimal research tool because SNA maps out relationship networks among different people in a social group context 
47 (Valente, Gallaher, \& Mouttapa, 2004). Additionally, utilizing SNA, researchers can describe the patterns of structural connectivity using a visual analysis of the networks or by generating statistical descriptions (Crnovrsanin et al., 2014). Therefore, SNA can help understand various risk behaviors that can be affected by other people (Smith \& Christakis, 2008) and can help researchers assess adolescents' risky behaviors within peer networks, as well as identify the structures of friendship ties that can influence behaviors.

In the U.S., over the last decade, researchers have studied peer effects upon adolescents' health risk behaviors using network structure data from the National Longitudinal Study of Adolescent Health to Adult Health (Add Health). The Add Health study gathers data on adolescents' health risk behaviors from a stratified sample of high schools (grades 7 - 12) nationwide, thus generating representative data. Furthermore, the Add Health data focus on social contexts (i.e., friendships and family relationships) that influence adolescents' health-related behaviors (Harris et al., 2009). Data are collected from in-school questionnaires and in-home interviews with adolescents, their peers, parents, and school administrators (Harris et al., 2009).

Several researchers have analyzed the Add Health data using social network analysis, demonstrating that SNA is a useful method for assessing both the structure of peer relationships, and/or friendship networks (Fujimoto \& Valente, 2012a; Mundt, 2011). These studies indicate that either friendship ties or peer effects among adolescents can function as causal factors directly influencing peers' risk behaviors such as drinking and smoking. Also, peer influences affect behaviors both positively and negatively, depending on adolescents' perceptions of friends' behaviors (Sieving et al., 2006). Despite its valuable contribution, research utilizing Add Health data varies in focus, with researchers examining many different types of friendships and various adolescent behaviors, such as drinking, tobacco use, and sexual intercourse. 
The purpose of this study, therefore, is to answer the following questions through systematically reviewing the extant literature: 1) Which risky health behaviors have been examined using SNA and the Add Health data?; 2) What findings have been identified in this literature relevant to friendship networks' impact on adolescents' risk behaviors?; and 3) What is the methodological quality of this body of literature?

Systematic literature reviews contribute to a body of literature by organizing and assessing scientific findings to effectively demonstrate both the accuracy and reliability of evidenced-based information (Mullen \& Ramirez, 2006; Mulrow, 1994). A long term goal of this review is to lend further validity to applying SNA as a method for studying adolescents' health-risk behaviors and assist future researchers in developing guidelines for implementing network-based intervention programs.

\section{Background}

National data, collected every two years by the YRBS and hosted by the Centers for Disease Control and Prevention (CDC), report that risky behaviors including tobacco use, drinking alcohol, and sexual intercourse at a young age have been health concerns for U.S. adolescents for more than 20 years. For instance, between 18 and 47\% of adolescents in grades 9 through 12 engaged in smoking, drinking alcohol, or were involved in sexual activity in 2011 (Centers for Disease Control and Prevention, 2012c).

These behaviors are the main health challenges for adolescents because continued risky behaviors are associated with increasing health problems. Previous studies have indicated that smoking and drinking alcohol at an early age can lead to poor health, an increased risk for alcoholism (Englund et al., 2008), and chronic diseases (e.g., cardiovascular and cancer) (Sawyer 
et al., 2007). Moreover, early sexual activity among adolescents can increase the risk of contracting sexually transmitted infections (STIs) (Kaestle et al., 2005) and the Human Immunodeficiency Virus (HIV) (Parillo et al., 2001).

In addition, researchers have reported that if adolescents are involved in a risk behavior, they are more likely to engage in different risk behaviors simultaneously. Johnson et al. (2000), for instance, identified a correlation between tobacco use and alcohol consumption among adolescents. Authors found adolescents who smoke are more likely to engage in binge drinking, simultaneously. Likewise, adolescents used to drinking heavily are more likely also to smoke regularly.

\section{Sexual behavior}

Adolescents who engage in unprotected sexual behaviors have a considerably higher risk of experiencing an unintended pregnancy or contracting STIs, including HIV (Tapert et al., 2001), than those who do not engage in these behaviors. As of 2011, the YRBS reported that the percentage of adolescents (grades 9 through 12) responding positively to the question "ever had sexual intercourse" was 47.4\% (Centers for Disease Control and Prevention, 2012d). Although this percentage is high, it represents a decline: in 1991, more than half (54.1\%) of adolescents in grades 9 through 12 reported that they had engaged in sexual intercourse.

Due to its many negative health and psychosocial consequences (not the least of which are sexual abuse and statutory rape), having had sexual intercourse before the age of 13 is another problematic behavior among adolescents. Rates for this behavior have plateaued between 2001 and 2009 (Centers for Disease Control and Prevention, 2012d), and have dropped 4\% (from $10.2 \%$ to $6 \%$ ) compared to 1991 . 
117 Alcohol use

118 In the United States, alcohol use by adolescents is illegal (under age 21) and remains a public

119 health problem also because it is associated with different risk behaviors, including tobacco use

120 and unprotected sexual intercourse. Data from the YRBS documents that, in 2011, an estimated

$12170.8 \%$ of adolescents reported they "ever had at least one drink of alcohol on at least 1 day"

122 during their lifetime (Centers for Disease Control and Prevention, 2012a). This statistic shows

123 the percentage fell $11 \%$ compared to 1991.

124 By $2011,38.7 \%$ of adolescents reported that they had "had at least one drink of alcohol

125 on at least 1 day" during the 30 days before the survey (Centers for Disease Control and

126 Prevention, 2012a). This percentage dropped from 50.8\% in 1991, a 12\% decrease.

\section{Tobacco use}

129 Smoking is related to morbidity and mortality, and is a leading cause of chronic diseases (e.g., 130 cardiac disease and vascular disease). Although smoking under the age of 18 years is illegal in 131 the U.S. (U.S. Food and Drug Administration, 2014), data from the YRBS in 2011 indicate that $13244.7 \%$ of teens reported they "had tried cigarette smoking" (Centers for Disease Control and 133 Prevention, 2012e). This rate has fallen by 25\% since 1991 (70.1\%). Another problematic 134 smoking behavior among adolescents (i.e., "smoked cigarettes on at least 1 day") when assessed 135 in 2011, indicated 18.1\% were smokers (Centers for Disease Control and Prevention, 2012e). 136 This rate has fallen 9.4\% since 1991 (from 27.5\%). While during the period $1991-1997$ the 137 rates had gradually increased to $36.8 \%$, the numbers have steadily decreased during 138 between1999 and 2011. 
Marijuana and Cocaine use

141 For adolescents, marijuana and cocaine use can cause unexplained changes in personality or

142 attitudes such as anxiety, poor social skills, interpersonal alienation, and poor impulse control.

143 These drugs also can affect physical development (e.g., brain and nerve damage, respiratory

144 problems, and blood pressure) (National Institute on Drug Abuse, 2014a; Brook, Balka, \&

145 Whiteman, 1999; Shedler \& Block, 1990; Volkow, Compton, \& Weiss, 2014). Moreover, they

146 can lead adolescents to other risky behaviors (e.g., sexual intercourse or drinking alcohol).

147 Marijuana and cocaine use are illegal for adolescents in the U.S. (National Institute on Drug

148 Abuse, 2014b), yet in 2011,39.9\% of adolescents reported, in the YRBS, ever using marijuana

149 "one or more times" (Centers for Disease Control and Prevention, 2012b). This rate has steadily

150 increased by $8.6 \%$ since 1991 . Between 1991 and 1999 the increase was even larger, from $31.3 \%$

151 to $47.2 \%$.

152 During that same time-period, an estimated $6.8 \%$ of adolescents reported they "ever used

153 any form of cocaine one or more times" (Centers for Disease Control and Prevention, 2012b).

154 Moreover, from 1991 to 1999 , the rates had slightly increased (5.9\% to $9.5 \%$ ).

\section{Methods}

157 To examine whether friendship networks on influence adolescents' risk behaviors in studies

158 utilizing SNA and Add Health data, we adopted Garrard's Matrix Method to search the literature

159 and qualitatively synthesize study findings (Garrard, 2010). We searched publications that

160 specifically used the Add Health data, catalogued by the website for the National Longitudinal

161 Study of Adolescent to Adult Health in Carolina Population Center at University of North 
162 Carolina at Chapel Hill. However, because the search engine in the website was limited, it

163 became necessary to identify additional articles through other electronic bibliographies. We

164 identified and retrieved, therefore, all peer-reviewed journal articles housed in three additional

165 electronic databases (Medline, Eric, and PsycINFO), and searched using variations of MeSH

166 terms combined with Boolean operators (e.g., sexual behavior, drinking behavior, adolescent,

167 and, social network, or network analysis). Additionally, we searched reference lists from each

168 study, for additional articles. Using the Scopus database, we conducted further searches based on

169 the first and corresponding author(s)' names listed in the retrieved reports.

170 Searching databases for this review initially yielded 4,455 results. Of these, 2,147 were

171 identified in the Add Health website, 2240 in Medline, and 68 in Eric and PsycINFO. After

172 identifying irrelevant topics and removing duplicates in an initial screening step, we identified 87

173 relevant studies. Among these, 73 were excluded based on our criteria. To be included in our

174 review studies needed to: (1) be published in a peer-reviewed journal between 2003 and 2014; (2)

175 be written in English; (3) use SNA to study friendship networks' influence on adolescents' risky

176 health behaviors; (4) focus on adolescents (aged 12 to 18 years old) in grades 7 through 12 (as

177 these are the grades utilized in the Add Health data); and 5) utilize the Add Health data. We

178 excluded studies if (1) only abstracts were published; (2) articles did not use SNA to study

179 adolescents' risky health behaviors; (3) studies employed SNA, but did not utilize the Add

180 Health data; (4) studies focused on the relationship between friendships and adolescents risky

181 behaviors; and (5) studies employed hypothetical models or simulation modeling to examine the

182 Add Health data.

183 Thus, we identified 14 articles eligible for full-text review. Moreover, we retrieved 1

184 additional article through retrieved studies' reference lists, and through first and corresponding 
185

186

187

188

author searches in Scopus. This study was published in 2001, but we included it in this review, because it met our other criteria. Finally, 15 articles met our inclusion criteria, and became the final sample in this review (see Figure 1 as an adapted PRISMA flow diagram) (Moher et al., 2009).

\section{Insert Figure 1 (PRISMA Flow diagram of reviewed studies) here}

Subsequently, we employed a review matrix to organize the information extracted from each article. The review matrix (see Appendix B) included information for each study on: authors, sample, focal variables (behaviors studied), purpose, use of theory, statistical analyses, key findings, and suggesting prevention/intervention programs.

We assessed each article's methodological quality employing a numerical score that reflects specific features of a study's design and analyses (Goodson, Buhi, \& Dunsmore, 2006; Jeon, Chen, \& Goodson, 2012). In this review, each study received a methodological quality score (MQS), reflecting its performance on the criteria outlined in Table 1, to include: whether studies examined a single or multiple risk behaviors; if studies utilized an established theoretical framework; if the report contained visualizations of the networks; if the report presented visualizations of the analysis; if the study tested specific hypothesis; if the report explained the types of data analysis employed; and whether researchers made recommendations for developing programs, based on their findings. The scores ranged from 1 to 9 with a higher value representing better methodological quality. 
207 Fifteen studies met our inclusion criteria. Most studies $(n=14)$ were conducted in the U.S., and

208 one paper, in France. Most reviewed studies $(\mathrm{n}=11)$ were published between 2009 and 2014,

209 perhaps because social network analysis only recently became popular as an analytical tool. Even

210 though network data were collected in Wave I of the Add Health survey (1994-1995), we found

211 the earliest publication on social networks among the reviewed studies was published in 2001.

212 All reviewed studies appeared in journals with impact factors ranging from 1.638 to

213 4.266. Four were published in the Journal of Adolescent Health, and two studies were published

214 in Addictive Behaviors. The other journals (the American Sociological Review, the American

215 Journal of Public Health, Health Psychology, Developmental Psychology, Social Science \&

216 Medicine, Academic Pediatrics, the Journal of Adolescence, the Journal of Health Economics,

217 and Perspectives on Sexual and Reproductive Health) published one report each.

218

219 Studies' findings

220 1) Which adolescents' risky health behaviors have been examined using SNA and the Add Health

221 data?

222 The studies in this review utilized SNA to examine adolescents' substance use — drinking,

223 smoking, and marijuana use - and sexual behavior — specifically, sexual intercourse.

224 Eight studies examined adolescents' alcohol consumption behaviors, focusing on

225 different aspects of adolescents’ drinking (Ali \& Dwyer, 2010; Clark \& Lohéac, 2007; Fujimoto

226 \& Valente, 2012a, 2012b, 2013; Jaccard, Blanton, \& Dodge, 2005; Kreager \& Haynie, 2011;

227 Mundt, 2011). Among these reports, six studied adolescents' drinking frequency as affected by

228 best (or close) friends, peer group, affiliated members (e.g., sports and club activities), or direct

229 (and indirect) friends (Ali \& Dwyer, 2010; Clark \& Lohéac, 2007; Fujimoto \& Valente, 2012a, 
230

231

232

233

234

235

236

237

238

239

240

241

242

243

244

2012b, 2013; Mundt, 2011). Moreover, two studies (Jaccard, Blanton, \& Dodge (2005), and Kreager and Haynie, 2011) investigated adolescents' level of drinking (specifically, bingeing) as influenced by friends.

In the six studies focused on drinking frequency, researchers used various questions from the Add Health questionnaires, including: "During the past 12 months, on how many days did you drink alcohol?" and "Think of all the times you have had a drink during the past 12 months, how many drinks did you usually have each time?"; "Over the past 12 months, on how many days have you gotten drunk or 'very, very high' on alcohol?"; and "During the past 12 months, how often did you get drunk?". In the two studies examining level of drinking (specifically,

bingeing), researchers used the following questions: "Over the past twelve months, on how many days did you drink five or more drinks in a row?" Both the adolescents and their friends were asked this question.

Seven studies focused on cigarette use or smoking behaviors among adolescents (Alexander et al., 2001; Ali \& Dwyer, 2009; Clark \& Lohéac, 2007; Fujimoto \& Valente, 2012a, 2012b; Lakon, Hipp, \& Timberlake, 2010; Pollard et al., 2010). All seven examined adolescents' frequency of smoking as influenced by various friendships, such as close or best friends, popular friends, mutual friends, or direct (and indirect) friends.

In these seven studies, authors used various questionnaire items, including: "During the past 30 days [past 12 months], on how many days did you smoke cigarettes?"; "During the past 30 days, on the days you smoked, how many cigarettes did you smoke each day?"; or "Of your 3 best friends, how many smoke at least 1 cigarette a day?”.

Three studies investigated sexual behavior (intercourse) (Ali \& Dwyer, 2011; Jaccard, Blanton, \& Dodge, 2005; Sieving et al., 2006). These studies examined the frequency of sexual 
253

254

255

256

257

258

259

260

261

262

263

264

265

266

267

268

269

270

271

272

273

274

275

intercourse as being influenced by close friends. Researchers used the questions: "Have you ever

had sexual intercourse?"; "In what month and year did you have sexual intercourse most

recently?"; or "If you had sexual intercourse, your friends would respect you more" from a section on "Motivations to Engage in Risky Behaviors".

Two studies (Clark \& Lohéac, 2007; Tucker et al., 2014) focused on adolescents’

marijuana use as influenced by peer groups. In this study, researchers used the questionnaire item:

"During the past 30 days, how many times did you use marijuana?" In these studies, authors examined the frequency of marijuana use as influenced by adolescents' friendships.

Even though the Add Health questionnaires have items addressing two different

behaviors in tandem (e.g., sexual intercourse + drinking; and sexual intercourse + drugs), none of the reviewed studies examined more than one behavior at a time.

2) What research findings have been identified in the literature relevant to friendship networks' impact on adolescents' risk behaviors?

Alcohol use

As mentioned previously, eight reviewed studies investigated the relationship between drinking alcohol and friendship networks (drinking frequency and amount of drinking). For instance, the study conducted by Fujimoto and Valente (2012a) examined how various friendship types influenced adolescents' substance use, including drinking (frequency). Authors classified three types of friendships: mutual friendships, directional friendships, and intimate friendships (see Figure 2 for diagrams of the three types of friendships examined by Fujimoto and Valente, 2012a). A mutual friendship was defined as reciprocated friends (knowing each other as friends). A directional friendship was defined as an unreciprocated nomination that originated either from 
276

277

278

279

280

281

282

283

284

285

286

287

288

289

290

291

292

293

294

an ego or from an alter (i.e., ego-nominating friend and alter-nominating friend). An intimate friendship was defined as closest or best friends who were being first nominated (Figure 2).

These three friendship types were based on friendship nominations that students were asked to make as they nominated five best male friends and five best female friends from the Add Health data.

\section{Insert Figure 2 (Diagrams of the three types of friendships examined by Fujimoto \& Valente} (2012a) here

Fujimoto and Valente (2012a) found mutual friends were more likely to influence their friends' drinking behavior (frequency) than a directional friendship in the previous year (AOR = 2.07; $p<0.001)$. Moreover, in the directional friendships among unreciprocated alters, the authors found ego-nominating friends (Figure 2) were slightly more influential in adolescents' drinking behavior than alter-nominating friends $(\mathrm{AOR}=2.02 ; p<0.001)$. Paradoxically, for the intimate relationships (Figure 2), the study indicated that non-best friends were more likely to influence adolescents' past year drinking than best friends $(\mathrm{AOR}=2.62 ; p<0.001)$.

Fujimoto and Valente (2013) also examined the influence on adolescents' drinking (and drinking frequency) of friends and affiliated members in sports and club activities. Adolescents were asked in which school organized clubs or sports they participated. Based on this information, authors divided activities into 12 types of sports and clubs, such as playing chess, studying French, and basketball.

Moreover, Fujimoto \& Valente (2013) categorized friendships as 1) all nominated friends (adolescent nominated the alter as a friend, the equivalent to "directional friendships" in Figure 2), and 2) only reciprocated friends (both adolescents mutually called each other friends) (Figure 2). Fujimoto \& Valente (2013) then created affiliation models based on nominated friends (i.e., 
299 General affiliation - the influence from all members' friendships; Nominated-friends'

300 affiliation - the influence from adolescents who were nominated as friends; and Nonfriends'

301 affiliation - the influence from adolescents who were not nominated friends). Affiliation models

302 based on reciprocated friends were also developed (i.e., General affiliation - the influence from

303 all members' friendships; Reciprocated-friends' affiliation — the influence from adolescents who

304 had at least one reciprocated friend; and Nonreciprocated-friends' affiliation - the influence of a 305 nonreciprocated friend).

306 In the nominated friends' general affiliation model, sports members influenced

307 adolescents' drinking and frequency of drinking ( $\mathrm{AOR}=1.20 ; p<0.05)$, and club members only

308 affected adolescents' drinking ( $\mathrm{AOR}=1.46 ; p<0.01)$. This study additionally demonstrated that

309 friends who drink were also more likely to affect adolescents' drinking and drinking frequency

$310(\mathrm{AOR}=1.55 ; p<0.001)$. In the nominated-friends' affiliation model, this study indicated club

311 members significantly influenced adolescents' drinking and drinking frequency ( $\mathrm{AOR}=1.15 ; p$

$312<0.01)$ (Fujimoto \& Valente, 2013). In the nonfriends' affiliation model, club members who

313 were not friends were more likely to affect drinking and drinking frequency of adolescents (AOR

$314=1.37 ; p<0.01)$. In the reciprocated friends' affiliation model, sports members who were mutual

315 friends with adolescents significantly influenced drinking and frequent drinking (AOR $=1.16 ; p$

$316<0.01)$. Moreover, club members who were mutual friends were more influential in adolescents'

317 drinking and frequency of drinking $(\mathrm{AOR}=1.22 ; p<0.001)$ than the results based on the

318 nominated-friends affiliation model $(p<0.01)$. Additionally, in the nonreciprocated friends'

319 affiliation model, club members significantly influenced drinking and drinking frequency of

320 adolescents $(\mathrm{AOR}=1.25 ; p<0.05)$ (Fujimoto \& Valente, 2013). 

influenced other adolescents' drinking behavior within the affiliation friendship networks. This

323

324

325 finding can be explained by the fact that club members do not need to be intimate friends to be connected to each other, because they share many common interests and behaviors, even if they are not friends.

In another study, Jaccard, Blanton, \& Dodge et al. (2005) evaluated how close friends influence adolescents' binge drinking. In this study, close friends were defined as those who were nominated by adolescents. Authors found a statistical significance in the behavioral similarity (binge drinking) between adolescents and their close friends ([unstandardized regression coefficient] $0.15 ; p<0.006$ ). Additionally, the study demonstrated that when adolescents' drinking behavior changed between Wave I (1995) and Wave II (1996) of data collection, their close friends' binge drinking also changed accordingly during the same time period ([unstandardized regression coefficient] $0.12 ; p<0.05)$.

The other five studies showed similar findings, indicating that friendships that matter, among adolescents, were more likely to exert influence upon adolescents' drinking behavior. For instance, among these five studies, Clark and Lohéac (2007) found that "if participation in drinking alcohol by the male peer group in the same school year increases by $25 \%$, the adolescent's probability of drinking alcohol increases 4.5\%" (p. 773). Likewise, the study by Ali and Dwyer (2010) showed that if the number of close friends who drink increased by $10 \%$, other adolescents' drinking would increase by $2 \%$. Authors also found "a $10 \%$ increase in drinking among grade-level peers... associated with a 4\% increase in individual drinking" (p. 340).

\section{Tobacco use}


344 Seven of the fifteen studies reported friendship influence on adolescents' frequency of smoking.

345 For instance, Ali and Dwyer (2009) categorized peer network as not only close friends who were

346 nominated by the adolescents, but also those who were classmates and others from the same

347 grade in school. A key finding from the study was that "having up to 25 percentage of close

348 friends as smokers increases the probability of smoking by $5 \% \ldots$ whereas being in a class

349 containing up to $25 \%$ smokers increases the likelihood of smoking by $10 \%$ " (p. 406).

350 In another study, Fujimoto and Valente (2012b) investigated the influence of peer

351 networks on adolescent's substance use (smoking cigarettes and drinking alcohol), based on

352 contagion mechanisms, in terms of cohesion and structural equivalence. Cohesion referred to

353 relationships within a network, for which there are direct ties or exchange of influence. Structural

354 equivalence referred to relationships among adolescents who occupy similar positions as others

355 within friendship networks (see Figure 3 for diagrams of cohesion and structural equivalence in a

356 network). Authors defined peers as those who were nominated by friends. In their analysis, they

357 utilized a network exposure model to assess both cohesion and structural equivalence measuring

358 peers' risk taking in terms of social distances (at four steps away from other adolescents -

359 friends of friends of friends of friends). The results indicated "the odds ratios for cohesion

360 exposures to smoking were statistically significant up to distance two (but not significant for

361 distances greater than two) with the highest magnitude at distance one $(\mathrm{OR}=1.50 ; \mathrm{p}<0.001)$,

362 followed by distance two $(\mathrm{OR}=1.40 ; \mathrm{p}<0.001)$ " (p. 1957).

\section{Insert Figure 3 (Diagrams of cohesion and structural equivalence in a network) here}

364 These findings suggest that direct or indirect friends (a friend or the friend of a friend)

365 were more likely to influence adolescents' smoking behavior than friends at distance three or

366 four (the friend(1)-of-a-friend(2)-of-a-friend(3), or the friend(1)-of-a-friend(2)-of-a-friend(3)-of- 
367 a-friend(4)). Moreover, the researchers found that for structural equivalence exposure to

368 smoking, “...exposure effects were statistically significant for all distances with the highest in

369 magnitude at distance one $(\mathrm{OR}=1.99 ; \mathrm{p}<0.001)$, followed by distance two $(\mathrm{OR}=1.83 ; \mathrm{p}<0.001)$,

370 distance three $(\mathrm{OR}=1.59 ; \mathrm{p}<0.001)$ and distance four $(\mathrm{OR}=1.59 ; \mathrm{p}<0.001)$ " (Fujimoto \&

371 Valente, 2012b, p. 1957). These findings suggest that adolescents, who were one and two steps

372 away in the network structure, were more likely to affect adolescents' smoking behavior than

373 adolescents at three or four steps away in terms of social distances.

374 The other five studies showed similar results, namely, that various close friendships, such

375 as best, popular, and mutual friends, were more likely to influence adolescents' smoking

376 behavior than non-close friends. For instance, Alexander et al. (2001) indicated that if

377 adolescents have best friends who are cigarette smokers, those adolescents' probability of

378 smoking increases two fold. Similarly, the study conducted by Pollard et al. (2010) demonstrated

379 that “... a greater number of one's best friends [who] smoked, and increases in the perceived

380 number of best friends who smoked over a one-year period, were associated with greater odds of

381 an adolescent being [a smoker]...” (p. 682).

382

383

Sexual intercourse

384 Three studies focused on how adolescents' friendships influence each other's sexual behavior 385 (specifically, intercourse). For instance, Sieving et al. (2006) classified close friends as those

386 who were being nominated. Close friends were based on friendship nominations by students who

387 were asked to nominate best male and female friends. Researchers found that “...for every $1 \%$

388 increase in sexually experienced friends at Wave I [1995], the odds that young people initiated

389 sex by Wave 2 [1996] increased by 1\%” (p. 17). 
In another study, Ali and Dwyer (2011) defined peer group as not only close friends who

391

392

393 were nominated by adolescents, but also those who were classmates and others from the same grade in school. In this study, they found that if the number of close friends initiating sex increased by $10 \%$, an adolescent's probability of initiating sex would also increase by $5 \%$. The third study (Jaccard, Blanton, \& Dodge et al., 2005), showed similar findings, indicating that close friends were more likely to exert influence on adolescents to engage in sex. They found "...of target individuals whose closest friends engaged in sexual activity across the two waves [Waves I and II], 56\% also engaged in sexual intercourse across the waves" (p. 141). All reviewed studies examining sexual behaviors examined single behaviors; examined two or more behaviors in tandem, such as sexual activity with alcohol consumption, even though the Add Health data contains this information.

\section{Marijuana use}

Among the15 studies reviewed, two examined the influence of friendship networks on adolescent's marijuana use. Tucker et al. (2014) examined how different types of friendships (i.e., friend reciprocity, friend popularity, and popularity difference) affected adolescents' marijuana use in two saturated schools samples (i.e., school 1 and 2) at Wave I and II. Friend reciprocity was defined as mutual friends (being nominated by each other as friends). Friend popularity was defined based on "the total number of friendship nominations received by a nominated friend at a given wave..." (p. 68). Popularity difference was defined as "the difference in number of friend nominations received...” (p. 68). Researchers found that two friendships (i.e., reciprocity and friend popularity) were more likely to influence adolescents' marijuana use. Regarding friend reciprocity, authors found "in school 1, there was a significant positive interaction between 
413 friends' influence on marijuana use and friend reciprocity....adolescents tended to adopt the

414 [marijuana] use behaviors of their mutual friends..." (p. 72). For friend popularity, the

415 researchers found that there was only statistical significance in school 2, indicating popular

416 friends were more likely to influence adolescents' marijuana use.

417 The second study, conducted by Clark and Lohéac (2007), examined peer group effects

418 on adolescents' alcohol, smoking and marijuana use. However, in this study, authors did not find

419 that friendships influenced adolescents' marijuana use.

\section{Insert Table 1 here}

421

422

423

424

425

426

427

428

429

430

431

432

433

434

435

\section{3) What is the methodological quality of this body of literature?}

As previously mentioned, we assigned a methodological quality score (MQS - with a possible range of 1 to 9 points) to each reviewed study. Table 1 presents the distribution of reviewed studies in terms of the MQS criteria. The average MQS was 4.6 ( $\mathrm{SD}=1.24)$, with actual scores ranging from 2 to 7 points. Among the 15 studies, seven (46.7\%) scored below 4.5. The main problems affecting studies scoring below average were lack of visuals for the networks and/or for the analyses.

Eleven reviewed studies (73.3\%) focused on studying a single behavior (most commonly, smoking or drinking). Four studies (26.7\%) analyzed two or more behaviors, such as alcohol and tobacco use, but each behavior was examined separately. None examined two or more risk behaviors simultaneously (e.g., sexual intercourse with drug or alcohol consumption).

Regarding using or adopting theoretical frameworks, while the majority (10 studies) employed a theoretical framework, five studies (33.3\%) failed to do so. Among the 10 studies employing a theoretical framework, six $(40 \%)$ provided some theoretical explanation or rationale and four studies (26.7\%) presented a scientific or behavioral theory: Social Learning Theory and 
436 Social Comparison Theory. Eight studies (53.3\%) did not test a hypothesis. Seven reports

$437(46.7 \%)$ tested a proposed hypothesis such as “....influence from mutual friendships has stronger

438 influence on adolescent drinking and smoking than non-mutual friendships" (Fujimoto \&

439 Valente, 2012, p. 137) or "adolescents with higher proportions of sexually experienced close

440 friends are more likely to initiate sexual intercourse than others" (Sieving et al., 2006, p. 14).

441 Only one reviewed study $(6.7 \%)$ provided visual graphics for the networks examined,

442 while four studies (26.7\%) provided illustrations for how friendship influences egos and their

443 alters. Fourteen studies (93.3\%) employed and reported both descriptive and inferential statistics

444 in their data analysis. One study (6.7\%) reported only inferential statistics. More than half of the

445 reviewed studies (66.7\%) made recommendations for prevention or intervention programs, based

446 on their network-related findings. Lakon, Hipp \& Timberlake (2010), for instance, propose in

447 their study: "These friendship pairs could be targeted for a school-based intervention, either to

448 help both adolescents in a pair remain nonsmokers or so that they could help each other stop

449 smoking" (p. 1226-1227).

450

451

\section{Discussion}

452 This systematic review consolidated a segment of the current research employing SNA for 453 studying adolescents' health risk behaviors. Specifically, we synthesized findings from network 454 analyses based on the Add Health data, and assessed each analysis' methodological quality 455 (presented in Table 1).

456 In this review, fifteen studies met our inclusion criteria. These studies found that, in 457 general, various types of friendships exert influence upon adolescents' health risk behaviors. 
458

459

460

461

462

463

464

465

466

467

468

469

470

471

472

473

474

475

476

477

478

479

480

Across reviewed studies, having friends engaging in risky behaviors is a negative predictor of adolescents' healthy behaviors or a positive predictor of risky ones.

More than half of the reviewed studies examined friendship network effects on

adolescents' risky behaviors either at a single point in time, or over time. Based on these studies,

we learn that individuals who have friends or are linked to friendship networks exhibiting risky

behaviors (e.g., smoking or alcohol consumption) are at increased risk for engaging in these

behaviors both initially, and over time.

These findings from the Add Health data mirror results from a longitudinal study

conducted in Finland. Mercken et al. (2012) assessed the relationship between substance use

(alcohol consumption) and friendship networks among Finnish adolescents at different data

points (i.e., time 1, time 2, time 3 , and time 4). The results demonstrated that friends with risky

drinking behaviors influenced adolescents to engage in similar drinking behaviors over time

(between time 1 and 2). These results indicate, therefore, that SNA can account for the role of

time in risky behaviors with more nuanced information than traditional longitudinal designs

(Rothenberg et al., 1998).

The reviewed studies highlighted that SNA can help researchers better understand the complex mechanisms underlying the connection between friendships among adolescents and risky behaviors. Even studies that utilize SNA but are not included in this review mention SNA is a helpful tool for understanding adolescent behaviors as an outcome of social relationships, as well as for understanding changes in behaviors and friendship networks over time (Christakis \& Fowler, 2007; Luke \& Harris, 2007; Mercken et al., 2009; Smith \& Christakis, 2008), because friendship ties and behaviors occur inside the structure of dynamic interpersonal relationships among adolescents (Steglich, Snijders, \& Pearson, 2010). For instance, adolescents may choose 
481 friends having similar behaviors as theirs, or they may change their behaviors to develop new

482 friendships or to match the behavior of existing friends. SNA, thus, can help explain peer

483 selection, as well as lead to modeling changes in behaviors as a function of ties over time

484 (Christakis \& Fowler, 2007; Luke \& Harris, 2007; Scott \& Carrington, 2011).

In addition, SNA also allows better understanding of phenomena that cannot be

486

487

488

489

490

491

492

493

494

495

496

497

498

499

500

501

502

503

adequately studied with traditional linear analyses. In particular, linear analysis cannot provide measures of structural linkages among individuals located inside a network, as a supplement to measures of an individuals' health risk behaviors. Using SNA, however, researchers are able to account for, and examine network dynamics and structure, such as density (i.e., the number of actual connections as a function of the total possible connections in a network) or degree (i.e., the number of ties, in and out, with other individuals in a network) (Valente, 2010), the impact of a network structure upon health behaviors, as well as the role of individuals as a function of their placement in the network. Moreover, SNA can create visualizations, depicting ties among individuals (Scott \& Carrington, 2011; Valente, 2010), showing how an individual's position may act as a mediator for positive or negative behavioral influences. For instance, the reviewed study carried by Kreager and Haynie (2011) found that "indirect ties to a drinking peer through a romantic partner are associated with significantly higher future drinking than is the drinking of more proximal friends or romantic partners" (p. 756).

When SNA is employed in the study of health behaviors, it can not only identify structural and relational factors associated with behavioral changes in individuals or groups, but also provide information that can be used for developing effective network-based intervention programs to reduce health risk behaviors. In a study conducted by Valente et al. (2007), for instance, the authors compared changes in adolescents' substance use (i.e., cigarette, alcohol, 
marijuana, and cocaine) between a control group receiving an evidenced-based prevention program and a network group receiving peer-leader intervention as a network prevention program. The results indicated that using a peer-leader program targeting the network was more effective in reducing substance use after a one-year follow-up assessment. When assessed for overall methodological quality, the mean MQS for the studies reviewed herein was 4.6, an indicator of good quality relative to our seven criteria (a theoretical range of 1-9 points). Although the body of evidence we reviewed exhibits good methodological quality, because scores fell above the theoretical mid-point of our scale, not supplying illustrative visualizations showing the connections among individuals in networks, the absence of theoretical frameworks, and not examining two or more behaviors in tandem, affected the overall quality of this body of research, vis-à-vis our criteria.

One common weakness was the absence of either graphs depicting the networks or visualizations that could help understand the proposed analyses. Providing visualizations can improve the clarity of, and highlight structural relationships within networks, more easily (Crnovrsanin et al., 2014). For instance, Mundt (2011) depicted a visual network of alcohol initiators and alcohol abstainers from their sample. The graphics the author provided help us understand not only the relationship among these adolescents, but guide us to a better understanding of the network measures themselves (i.e., differences between networks' density and degree are made more meaningful, when accompanied by a visual aid).

Another methodological weakness we identified was the reviewed studies' lack of a theoretical framework to examine adolescents' risky behaviors. The absence of a theoretical framework in research can lead to overlooking of salient factors and examining spurious ones. Conversely, using a theoretical framework can facilitate identifying possible causes (Goodson, 
527 2010; Green, 2000). Theory helps develop programs, and findings from studies that use theory

528 can be useful for determining the type of intervention that best suits risky behaviors.

529 Understanding of social networks is growing, based on the increasing amounts of data being

530 collected. Nonetheless, in order to develop effective interventions that target adolescents'

531 networks, theoretical explanations of the mechanisms affecting behaviors within a network

532 become even more important. Using available theories of networks, adolescent development, and

533 structural influences on behavior, researchers can shed light upon the data they are now

534 collecting and, over time, build the knowledge-base on this topic.

535 A further weakness of the reviewed studies was examining behaviors, individually.

536 Studies focusing on two or more behaviors in tandem would allow for a better holistic

537 understanding of the role of friendship networks in the dynamics of adolescents' risky behaviors, 538 given that risk behaviors rarely happen in isolation. There is abundant evidence documenting 539 adolescents' engagement in multiple risky behaviors carried out simultaneously. In the review 540 conducted by Cooper (2002), for instance, the author found that college students who drink 541 alcohol were also involved in having sexual intercourse. Similar to Cooper's review (2002), a 542 study from Johnson et al. (2000) also identified that when teens engage in high levels of alcohol 543 consumption, they also were more likely to smoke.

544 Similar to the reviewed studies, this review also carries important limitations. First, 545 despite our attempt to locate all studies employing SNA utilizing the Add Health data, it is 546 possible our search did not capture all existing studies, given we limited the search to published 547 reports. Second, to assess the methodological quality of this literature, we adopted and created 548 the MQS criteria based on previous systematic reviews. The precise criteria we use in this review, 
549 therefore, have not been tested for their ability to generate valid and reliable assessments and 550 could, therefore, be biased.

551 Despite these limitations, this systematic review demonstrated the important role of

552 friends and friendship networks on adolescents' risky behaviors and the benefit of a SNA 553 approach for better understanding of this role and its complex mechanisms. Moreover, this

554 review is unique not only because it lends further validity to SNA as a method, but also because 555 it synthesizes findings from high quality studies based on a national sample. Identifying how 556 friendships or friendship networks function as pathways for adopting risky behaviors can also

557 help health educators and promoters to design guidelines for network intervention programs to 558 reduce adolescents' risky behaviors. 
561 Alexander, C., Piazza, M., Mekos, D., \& Valente, T. W. 2001. Peers, schools, and adolescent cigarette smoking. Journal of Adolescent Health 29(1):22-30. DOI:10.1016/S1054$139 X(01) 00210-5$

564

565

566

567

568

569

570

571

572

573

574

575

576

577

578

579

580

581

Ali, M. M., \& Dwyer, D. S. 2009. Estimating peer effects in adolescent smoking behavior: a longitudinal analysis. Journal of Adolescent Health 45(4):402-408. DOI:10.1016/j.jadohealth.2009.02.004

Ali, M. M., \& Dwyer, D. S. 2010. Social network effects in alcohol consumption among adolescents. Addictive Behaviors 35(4):337-342. DOI:10.1016/j.addbeh.2009.12.002

Ali, M. M., \& Dwyer, D. S. 2011. Estimating peer effects in sexual behavior among adolescents. Journal of Adolescence 34(1):183-190. DOI:10.1016/j.adolescence.2009.12.008

Brook, J. S., Balka, E. B., \& Whiteman, M. 1999. The risks for late adolescence of early adolescent marijuana use. American Journal of Public Health 89(10):1549-1554. DOI:10.2105/AJPH.89.10.1549

Centers for Disease Control and Prevention. 2012a. Trends in the prevalence of alcohol use National YRBS: 1991-2011. Available at http://www.cdc.gov/healthyyouth/yrbs/pdf/us_alcohol_trend_yrbs.pdf(accessed May 18 2013).

Centers for Disease Control and Prevention. 2012b. Trends in the prevalence of marijuana, cocaine, and other illegal drug use National YRBS: 1991-2011. Available at http://www.cdc.gov/healthyyouth/yrbs/pdf/us_drug_trend_yrbs.pdf(accessed May 19, 2013). 
582 Centers for Disease Control and Prevention. 2012c. Trends in the prevalence of selected risk

583

584

585

586

587

588

589

590

591

592

593

594

595

596

597

598

599

600

601

602

603

604

behaivors and obesity for all students National YRBS: 1991-2011. Available at http://www.cdc.gov/healthyyouth/yrbs/pdf/us_summary_all_trend_yrbs.pdf(accessed May 15, 2013).

Centers for Disease Control and Prevention. 2012d. Trends in the prevalence of sexual behaviors and HIV testing National YRBS: 1991 - 2011. Available at http://www.cdc.gov/healthyyouth/yrbs/pdf/us_sexual_trend_yrbs.pdf(accessed March 28, 2013).

Centers for Disease Control and Prevention. 2012e. Trends in the prevalence of tobacco use National YRBS: 1991-2011. Available at http://www.cdc.gov/healthyyouth/yrbs/pdf/us_tobacco_trend_yrbs.pdf(accessed May 18, 2013).

Christakis, N. A., \& Fowler, J. H. 2007. The Spread of obesity in a large social network over 32 years. New England Journal of Medicine 357(4):370-379. DOI: doi:10.1056/NEJMsa066082

Clark, A. E., \& Lohéac, Y. 2007. "It wasn't me, it was them!" social influence in risky behavior by adolescents. Journal of Health Economics 26(4):763-784. DOI:10.1016/j.jhealeco.2006.11.005

Cooper, M. L. 2002. Alcohol use and risky sexual behavior among college students and youth: evaluating the evidence. Journal of Studies on Alcohol and Drugs (s14):101-117.

Crnovrsanin, T., Muelder, C. W., Faris, R., Felmlee, D., \& Ma, K. L. 2014. Visualization techniques for categorical analysis of social networks with multiple edge sets. Social Networks 37(0), 56-64. DOI:10.1016/j.socnet.2013.12.002 
605 Englund, M. M., Egeland, B., Oliva, E. M., \& Collins, W. A. 2008. Childhood and adolescent 606 predictors of heavy drinking and alcohol use disorders in early adulthood: a longitudinal 607 developmental analysis. Addiction 103(s1):23-35. DOI:10.1111/j.1360$608 \quad 0443.2008 .02174 . x$

609 Ennett, S. T., Bauman, K. E., Hussong, A., Faris, R., Foshee, V. A., Cai, L., \& DuRant, R. H. 2006. The Peer context of adolescent substance use: findings from social network 611 analysis. Journal of Research on Adolescence 16(2):159-186. DOI:10.1111/j.1532612 7795.2006.00127.x

613 Fujimoto, K., \& Valente, T. W. 2012a. Decomposing the components of friendship and friends' 614 influence on adolescent drinking and smoking. Journal of Adolescent Health 51(2):136615 143. DOI:10.1016/j.jadohealth.2011.11.013

616 Fujimoto, K., \& Valente, T. W. 2012b. Social network influences on adolescent substance use:

Garrard, J. 2010. Health Sciences Literature Review Made Easy: The Matrix Method, 3rd ed. Sudbury, MA: Jones and Barlett Publisher.

624 Goodson, P., Buhi, E. R., \& Dunsmore, S. C. 2006. Self-esteem and adolescent sexual behaviors, 625 attitudes, and intentions: a systematic review. Journal of Adolescent Health 38(3):310626 319. DOI:10.1016/j.jadohealth.2005.05.026 
627 Goodson, P. 2010. Theory in Health Promotion Research and Practice: Thinking Outside the 628 Box. Boston: Jones \& Bartlett Learning.

629 Green, J. 2000. The role of theory in evidence-based health promotion practice. Health $630 \quad$ Education Research 15(2):125-129. DOI:10.1093/her/15.2.125

631 Haas, S. A., Schaefer, D. R., \& Kornienko, O. 2010. Health and the structure of adolescent social 632 networks. Journal of Health Social Behavior 51(4):424-439.

633 DOI:10.1177/0022146510386791

634 635 636 637 638

Hall, J. A., \& Valente, T. W. 2007. Adolescent smoking networks: The effects of influence and selection on future smoking. Addictive Behaviors 32(12):3054-3059. DOI:10.1016/j.addbeh.2007.04.008

Harris, K. M., Halpern, C. T., Whitsel, E. A., Hussey, J., Tabor, J., Entzel, P. P., \& Udry, J. R. 2009. The National Logitudinal Study of Adolescent Health: Research Design. Available at http://www.cpc.unc.edu/projects/addhealth/design (accessed March 17, 2013).

Jaccard, J., Blanton, H., \& Dodge, T. 2005. Peer influences on risk behavior: an analysis of the effects of a close friend. Developmental Psychology 41(1):135-147. DOI:10.1037/00121649.41 .1 .135

Jeon, K. C., Chen, L. S., \& Goodson, P. 2012. Decision to abort after a prenatal diagnosis of sex chromosome abnormality: a systematic review of the literature. Genetics in Medicine 14(1):27-38. DOI:10.1097/GIM.0b013e31822e57a7

Johnson, P. B., Boles, S. M., Vaughan, R., \& Kleber, H. D. 2000. The co-occurrence of smoking and binge drinking in adolescence. Addictive Behaviors 25(5):779-783. DOI:10.1016/S0306-4603(99)00066-0 
649 Kaestle, C. E., Halpern, C. T., Miller, W. C., \& Ford, C. A. 2005. Young age at first sexual 650 intercourse and sexually transmitted infections in adolescents and young adults. American Journal of Epidemiology 161(8):774-780. DOI:10.1093/aje/kwi095

652

653

654

655

656

657

658

659

660

661

662

663

664

665

666

667

668

669

670

Kreager, D. A., \& Haynie, D. L. 2011. Dangerous liaisons? dating and drinking diffusion in adolescent peer networks. American Sociological Review 76(5):737-763. DOI:10.1177/0003122411416934

Lakon, C. M., Hipp, J. R., \& Timberlake, D. S. 2010. The social context of adolescent smoking: a systems perspective. American Journal of Public Health 100:1218-1228. DOI:10.2105/AJPH.2009.167973

Luke, D. A., \& Harris, J. K. 2007. Network analysis in public health: history, methods, and applications. Annual Review of Public Health 28(1):69-93.

DOI:10.1146/annurev.publhealth.28.021406.144132

Mercken, L., Snijders, T. A., Steglich, C., \& de Vries, H. 2009. Dynamics of adolescent friendship networks and smoking behavior: social network analyses in six European countries. Social Science \& Medicine 69(10):1506-1514.

DOI:10.1016/j.socscimed.2009.08.003

Mercken, L., Steglich, C., Knibbe, R., \& Vries, H. 2012. Dynamics of friendship networks and alcohol use in early and mid-adolescence. Journal of Studies on Alcohol and Drugs 73(1), 99-110.

Moher, D., Liberati, A., Tetzlaff, J., Altman, D. G., \& The PRISMA Group. 2009. Preferred reporting items for systematic reviews and meta-analyses: the PRISMA statement. PLoS Medicine 6(7):e1000097. DOI:10.1371/journal.pmed.1000097 
671 Mullen, P. D., \& Ramirez, G. 2006. The promise and pitfalls of systematic reviews. Annual 672 Review of Public Health 27:81-102. DOI:10.1146/annurev.publhealth.27.021405.102239

673 Mulrow, C. D. 1994. Rationale for systematic reviews. British Medical Journal 309(6954):597599. DOI:10.1136/bmj.309.6954.597

Mundt, M. P. 2011. The impact of peer social networks on adolescent alcohol use initiation. Academic Pediatrics 11(5):414-421. DOI:10.1016/j.acap.2011.05.005

677 National Institute on Drug Abuse. 2014a. DrugFacts: Marijuana. Available at http://www.drugabuse.gov/publications/drugfacts/marijuana (accessed August 23, 2014).

679

680

681

682

683

684

685

686

687

688

689

690

691

692

National Institute on Drug Abuse. 2014b. What is marijuana? Available at http://teens.drugabuse.gov/drug-facts/marijuana (accessed August 28, 2014).

Parillo, K. M., Freeman, R. C., Collier, K., \& Young, P. 2001. Association between early sexual abuse and adult HIV-risky sexual behaviors among community-recruited women. Child Abuse \& Neglect 25(3):335-346. DOI:10.1016/S0145-2134(00)00253-2

Pollard, M. S., Tucker, J. S., Green, H. D., Kennedy, D., \& Go, M. H. 2010. Friendship networks and trajectories of adolescent tobacco use. Addictive Behaviors 35(7):678-685. DOI:10.1016/j.addbeh.2010.02.013

Prinstein, M. J., Brechwald, W. A., \& Cohen, G. L. 2011. Susceptibility to peer influence: Using a performance-based measure to identify adolescent males at heightened risk for deviant peer socialization. Developmental Psychology 47(4):1167-1172. DOI:10.1037/a0023274

Rew, L., \& Horner, S. D. 2003. Youth resilience framework for reducing health-risk behaviors in adolescents. Journal of Pediatric Nursing 18(6):379-388. DOI:10.1016/S0882$5963(03) 00162-3$ 
693 Rothenberg, R. B., Sterk, C., Toomey, K. E., Potterat, J. J., Johnson, D., Schrader, M., \& Hatch,

694 S. 1998. Using social network and ethnographic tools to evaluate syphilis transmission.

695 Sexually Transmitted Diseases 25(3):154-160. DOI:10.1097/00007435-199803000-

6960009

697 Sawyer, S. M., Drew, S., Yeo, M. S., \& Britto, M. T. 2007. Adolescents with a chronic condition:

698 challenges living, challenges treating. Lancet 369(9571):1481-1489.

699 DOI:10.1016/S0140-6736(07)60370-5

700

701

Scott, J., \& Carrington, P. J. 2011. The SAGE Handbook of Social Network Analysis. London;

Thousand Oaks: SAGE Publications.

702

703

Shedler, J., \& Block, J. 1990. Adolescent drug use and psychological health. A longitudinal inquiry. American Psychologist 45(5):612-630. DOI:10.1037/0003-066X.45.5.612

Sieving, R. E., Eisenberg, M. E., Pettingell, S., \& Skay, C. 2006. Friends' influence on 705 adolescents' first sexual intercourse. Perspectives on Sexual and Reproductive Health 38(1):13-19. DOI:10.1363/psrh.38.013.06

Smith, K. P., \& Christakis, N. A. 2008. Social networks and health. Annual Review of Sociology 34(1):405-429. DOI:10.1146/annurev.soc.34.040507.134601

Steglich, C., Snijders, T. A. B., \& Pearson, M. 2010. Dynamic networks and behavior: Separating selection from influence. Sociological Methodology 40(1):329-393. DOI:10.1111/j.1467-9531.2010.01225.x

Tapert, S. F., Aarons, G. A., Sedlar, G. R., Brown, S. A. 2001. Adolescent substance use and sexual risk-taking behavior. Journal of Adolescent Health 28(3):181-189. DOI:10.1016/S1054-139X(00)00169-5 
715 Tucker, J. S. de la Haye, K, Kennedy, D. P., Green, H. D., \& Pollard, M. S. 2014. Peer influence 716 on marijuana use in different types of friendships. Journal of Adolescent Health 54(1):67-

717

718

719

720

721

722

723

724

725

726

727

728

729

730

731

732

733

734

735

736

737
73. DOI:10.1016/j.jadohealth.2013.07.025

U.S. Food and Drug Administration. 2014. Youth \& Tobacco. Available at http://www.fda.gov/TobaccoProducts/protectingkidsfromtobacco/default.htm (accessed August 28, 2014).

Valente, T. W. 2010. Social Networks and Health: Models, Methods, and Applications: Oxford University Press, USA.

Valente, T. W., Gallaher, P., \& Mouttapa, M. 2004. Using social networks to understand and prevent substance use: a transdisciplinary perspective. Substance Use \& Misuse 39(1012):1685-1712. DOI:10.1081/JA-200033210

Valente, T. W., Ritt-Olson, A., Stacy, A., Unger, J. B., Okamoto, J., \& Sussman, S. 2007. Peer acceleration: effects of a social network tailored substance abuse prevention program among high-risk adolescents. Addiction 102(11):1804-1815. DOI:10.1111/j.13600443.2007.01992.x

Volkow, N. D., Compton, W. M., \& Weiss, S. R. 2014. Adverse health effects of marijuana use. New England Journal of Medicine 371(9):879. DOI:10.1056/NEJMc1407928 


\section{Table $\mathbf{1}$ (on next page)}

Table 1. Methodological characteristics and frequency distribution of each criterion among 15 reviewed studies using Social Network Analysis and Add Health data 
2 Table 1. Methodological characteristics and frequency distribution of each criterion among 15

3 reviewed studies using social network analysis and Add Health Data

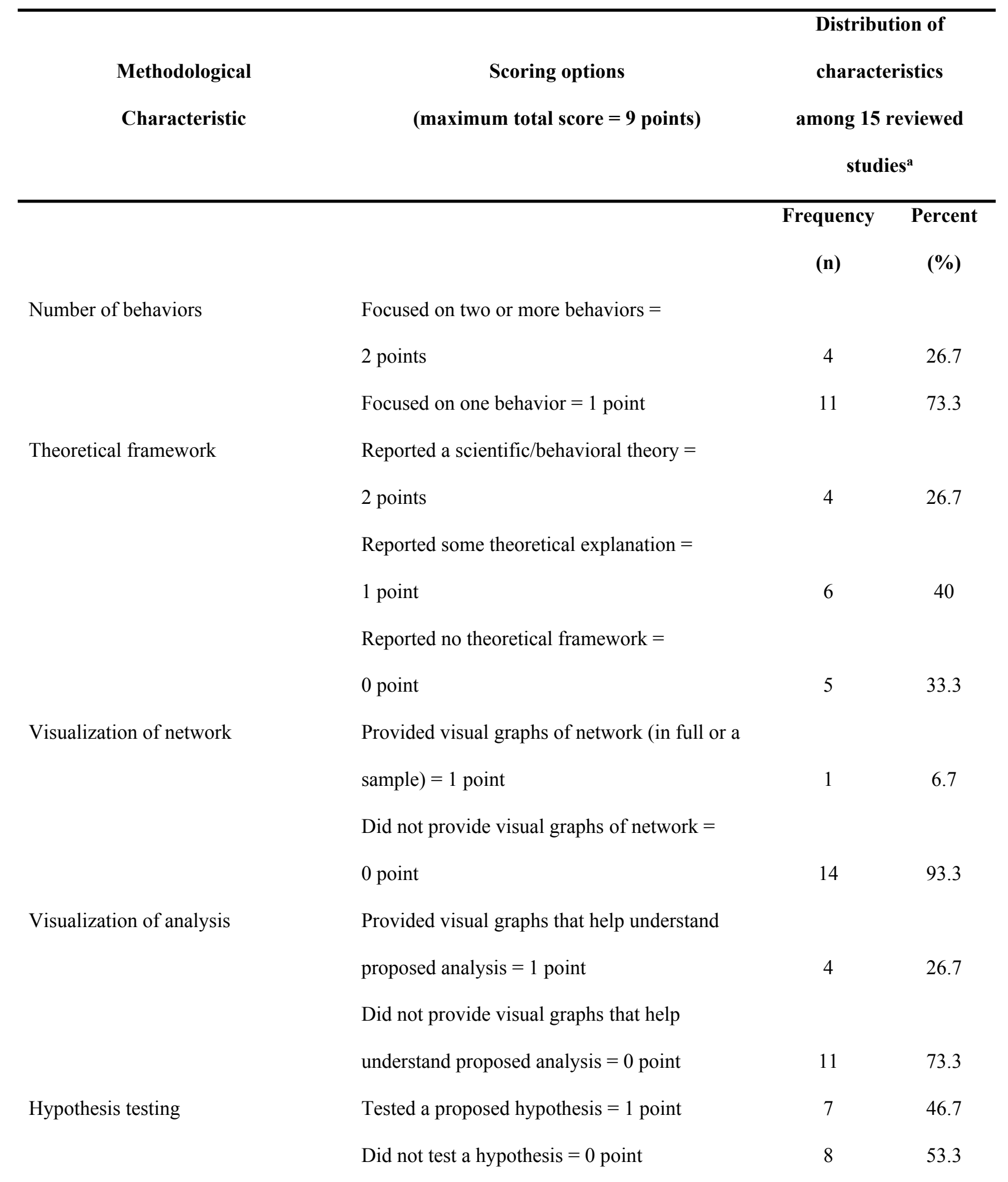


Data analysis

$\begin{array}{ll} & \text { statistics }=1 \text { point } \\ & \text { Reported only inferential statistics }= \\ & 0 \text { point } \\ \text { Recommendations for developing } & \text { Makes recommendations for } \\ \text { programs } & \text { prevention/intervention programs }=1 \text { point } \\ & \text { Makes no recommendations for developing } \\ & \text { programs }=0 \text { point } \\ & \text { Total possible maximum points }=9\end{array}$

14

93.3

1

10

5

$4.6(\mathrm{SD}=1.24)$;

actual range (2-7 points) 
Figure 1 (on next page)

Figure 1. PRISMA Flow diagram of reviewed studies 
Figure 1. PRISMA Flow diagram of reviewed studies

Search results from 2003 to 2014 :

Add Health website $(n=2,147)$ \& Medline $(n=2,240)$, Eric \& PsycINFO $(n=68)$,

Total $(n=4,455)$

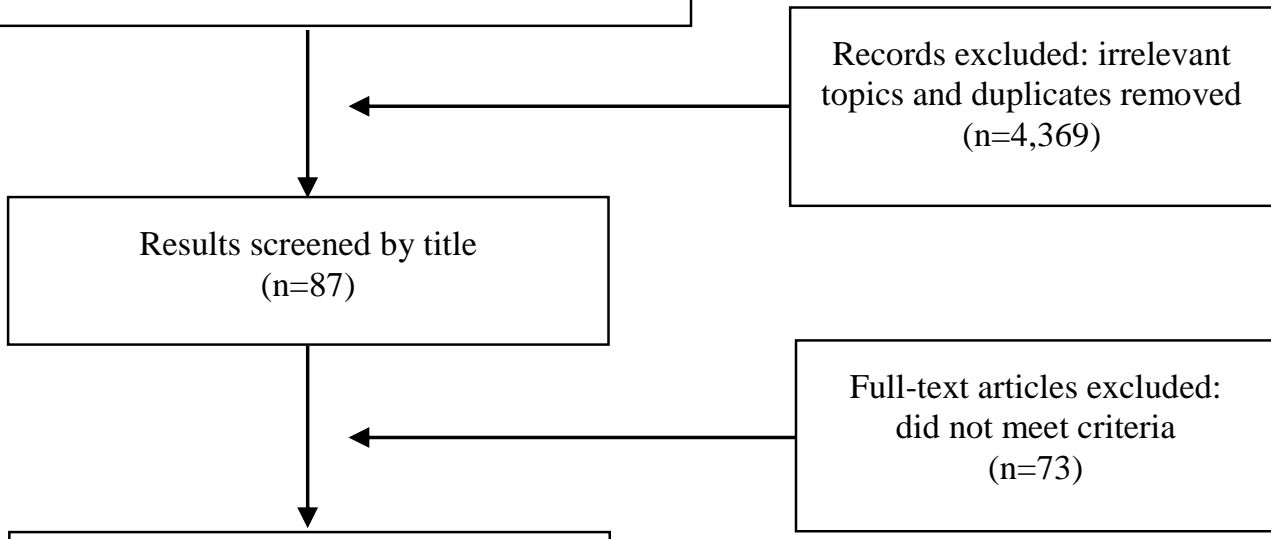

Full-text articles assessed for eligibility according to inclusion criteria $(n=14)$

Studies' reference lists, and first and corresponding authors through Scopus $(n=1)$

FINAL SAMPLE $(n=15)$ 
Figure 2 (on next page)

Figure 2. Diagrams of the three types of friendships examined by Fujimoto $\&$ Valente (2012a)

(A) Mutual/Reciprocated friendships. (B) Directional friendships: *Outdegree is the number of friendship ties that the ego who is a focal point within a network "sends" \& *Indegree is the number of friendship ties that the ego "receives" (Hall \& Valente, 2007). (C) Intimate friendships: *B was nominated as best or close friends by $\mathrm{A} ; \mathrm{C}-\mathrm{F}$ were nominated as friends, but not best or close friends. 
1 Figure 2. Diagrams of the three types of friendships examined by Fujimoto \& Valente

2 (2012a)

3

4 A

5

6

7

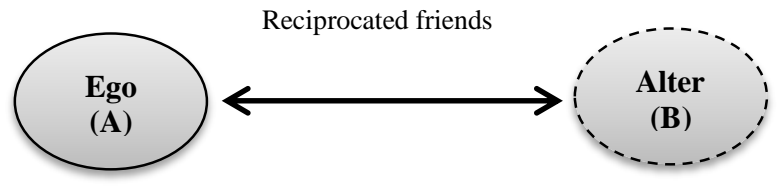

8

9

10

11

12 B

13

14

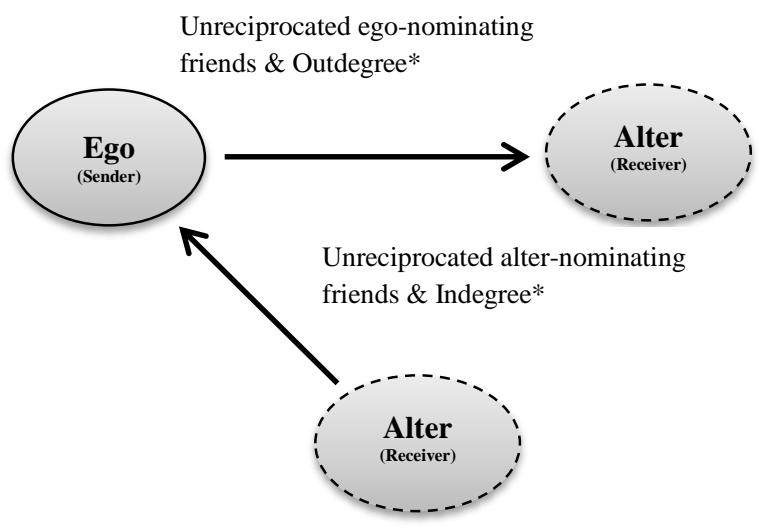

19

20

21 
$22 \mathrm{C}$

23

24

25

26

27

28

29

30

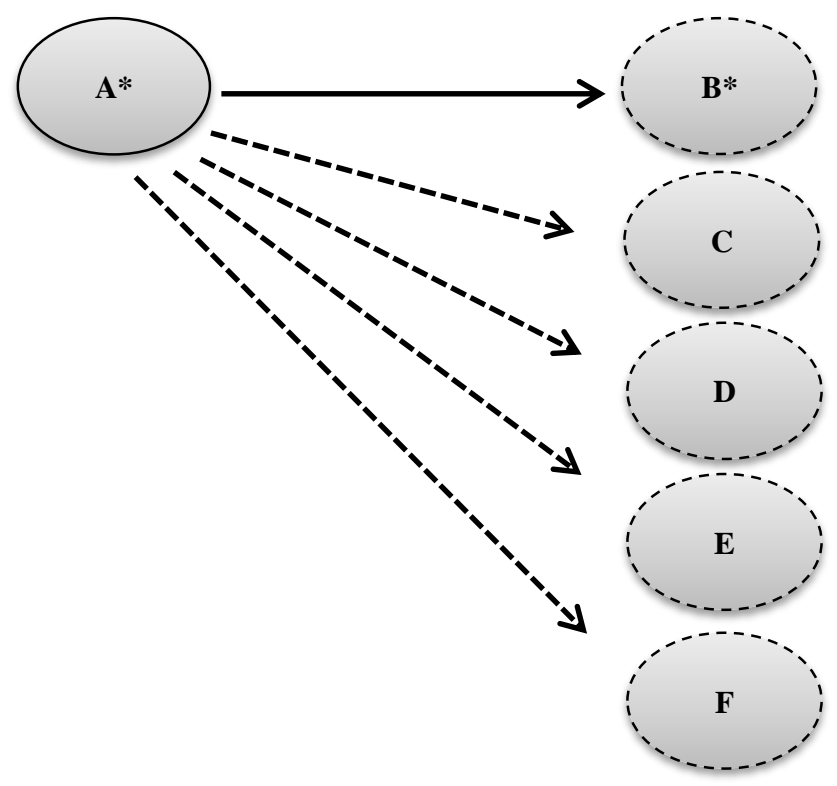

31 
Figure $\mathbf{3}$ (on next page)

Figure 3. Diagrams of cohesion and structural equivalence in a network

(A) Cohesion: ${ }^{*} \mathrm{C}$ has a direct tie with $\mathrm{A}$ and is influenced by $\mathrm{A}$. The relationships between $\mathrm{A}$ $B$ and $A-D$ are not cohesive, because the ties are indirect and there is no exchange of influence. (B) Structural equivalence: *B - C and C - D are structurally equivalent ties, because the individuals occupy the same position in the network. 
Figure 3. Diagrams of cohesion and structural equivalence in a network

A

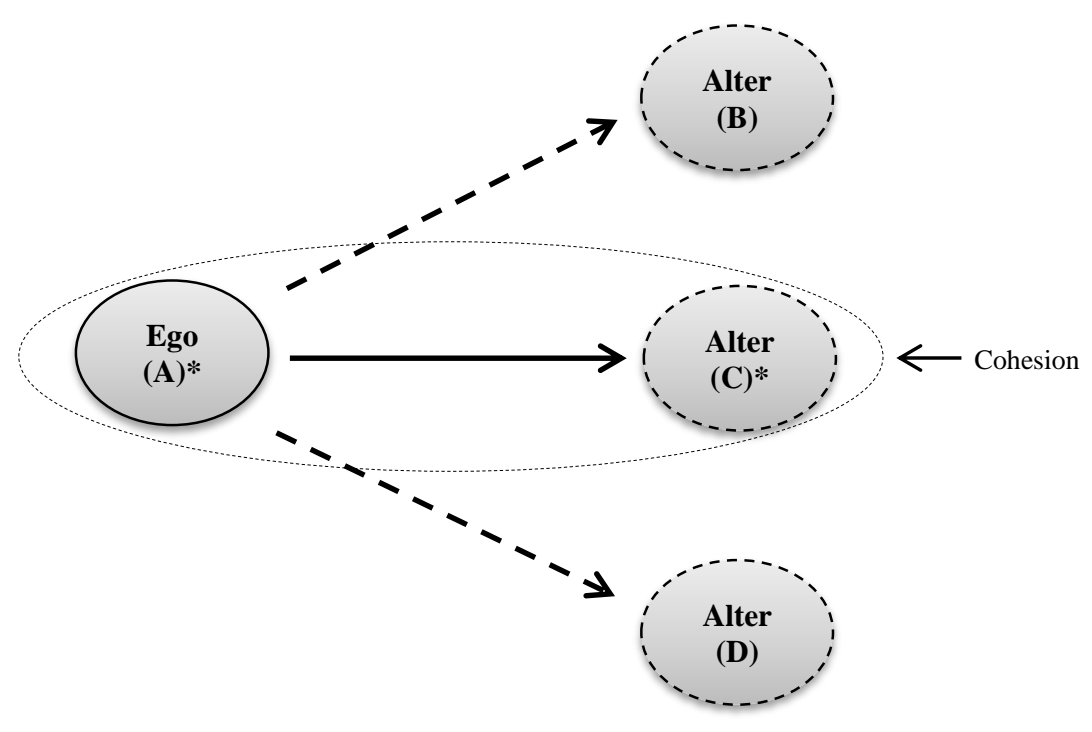

B

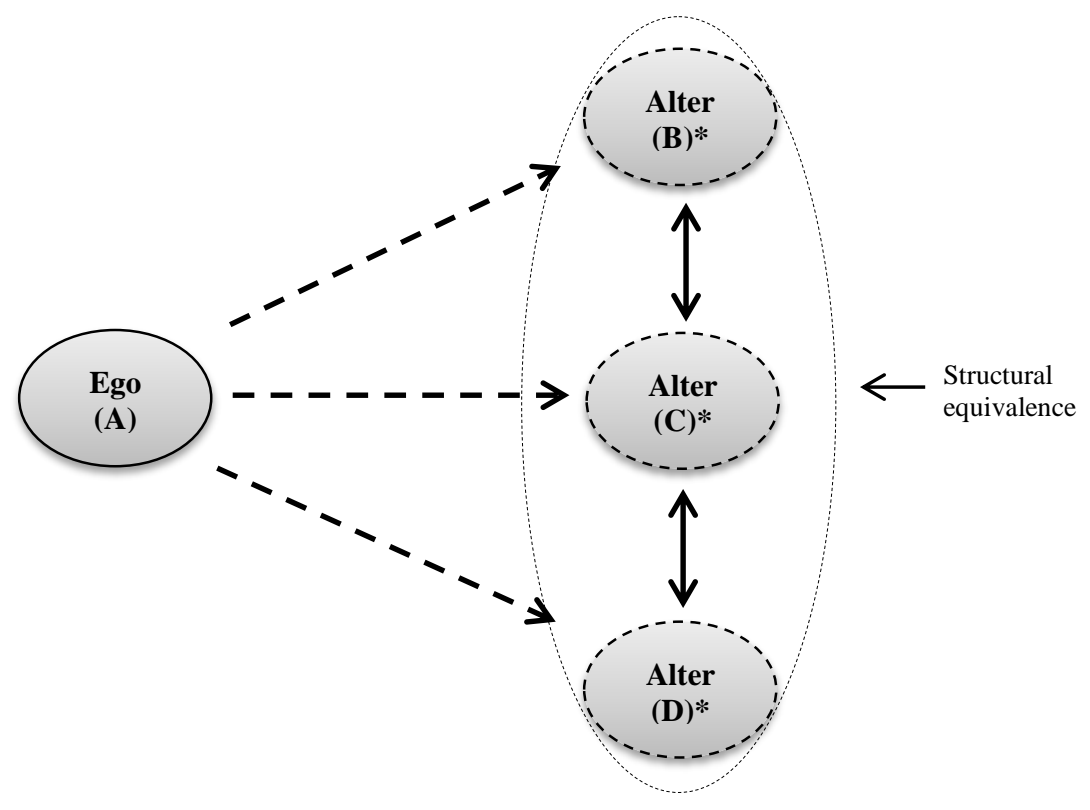

